\title{
Numerical analysis of streamline diffusion method for compressible flows
}

\author{
Jae Ryong Kweon*
}

(Received 16 February 1998; revised 9 February 1999)

\begin{abstract}
We study a finite element method for the linearized stationary compressible viscous Stokes system in a bounded domain with extra streamline diffusion added to the continuity equation. The differential equations to be considered are$$
\left\{\begin{array}{l}
-\mu \Delta \mathbf{u}-\nu \nabla \operatorname{div} \mathbf{u}+\rho(\mathbf{U} \cdot \nabla) \mathbf{u}+\nabla p=\mathbf{f} \quad \text { in } \Omega \\
\rho \operatorname{div} \mathbf{u}+\rho^{\prime} \mathbf{U} \cdot \nabla p=g \quad \text { in } \Omega,
\end{array}\right.
$$

*Department of Mathematics, College of Natural Science, Kyungpook National University, Taegu 702-701, KoREA. kweon@bh.knu.ac.kr

${ }^{0}$ See http://jamsb.austms.org.au/V41/E018 for this article and ancillary services, (C) Austral. Mathematical Soc. 2000. Published 19 May 2000, last corrected May 19, 2000.
\end{abstract}


Here, $\mathbf{U}$ is a given ambient fluid velocity vector and $\rho=\rho(P)$ is the density function of a given ambient fluid pressure $P$, and depending on the vector field $\mathbf{U}$, two different problems are considered. The unique existence and convergence analysis of the corresponding approximate solution is shown and numerical examples are given.

\section{Contents}

1 Introduction

2 The case with $\mathrm{U}=0$ on $\Gamma$

E34

3 Incoming compressible flow

E42

4 Numerical examples

E44

References

E49

\section{Introduction}

In this paper we study a finite element method (streamline diffusion method) for the compressible Stokes equations and give a convergence analysis. The differential equations considered are obtained by the linearization of the sta- 
tionary barotropic viscous compressible Navier-Stokes equations around an ambient flow (for the derivation of the equations see $[8,10]$ ). The steadystate barotropic compressible viscous Navier-Stokes equations are a system of PDEs of mixed type; the momentum equations form an elliptic subsystem in the velocity components, and the continuity equation is a hyperbolic equation in the density. Consequently the linearization of the system is also of mixed type.

The boundary conditions associated with the linearized and compressible Stokes system depend on the boundary values of the ambient flow vector field $\mathbf{U}$. If $\mathbf{U} \neq 0$ on the boundary, boundary values for the pressure are required on the incoming portion of the boundary. A discretization with optimal order convergence was obtained in [12]. This is also considered in Section 3. If $\mathbf{U}=0$ on the boundary, no boundary values for the pressure are required. A discretization of this problem was given in [8], with a nonoptimal error bound. Our purpose is to improve the result given in [8] by using the streamline diffusion method in the finite element formulation. The equations to be studied in this paper are given by

$$
\left\{\begin{array}{l}
-\mu \Delta \mathbf{u}-\nu \nabla \operatorname{div} \mathbf{u}+\rho(\mathbf{U} \cdot \nabla) \mathbf{u}+\nabla p=\mathbf{f} \quad \text { in } \Omega \\
\operatorname{div} \mathbf{u}+\kappa \mathbf{U} \cdot \nabla p=\rho^{-1} g \quad \text { in } \Omega, \\
\mathbf{u}=0 \text { on } \Gamma
\end{array}\right.
$$

where $\Omega$ is an open bounded domain with a smooth boundary $\Gamma$. Here $\mathbf{u}=[u, v]$ is a velocity vector, $p$ is the pressure; $\mathbf{U}=[U, V]$ is a given ambient fluid velocity vector with zero boundary value, $P$ is a pressure of ambient fluid, $\rho=\rho(P)$ is a given positive increasing function that is the 
density function of pressure, and $\kappa=\rho^{\prime} / \rho, \rho^{\prime}=d \rho / d P$. It is noted that $\kappa$ is positive. The functions $\mathbf{f}$ and $g$ are given, and the numbers $\mu$ and $\nu$ are the viscous constants with $\mu>0$ and $\mu>-\nu$. Regarding the ambient fluid, it is assumed that $U, V$, and $P$ are $C^{1}$ functions in the closure of $\Omega$.

It is well-known that there is a stable finite element method for the Stokes problem which gives a unique solvability and an optimal order convergence by establishing the inf-sup condition (see [2]). It is question if a similar result for the compressible Stokes system with $\mathbf{U}=0$ on $\Gamma$ can be obtained.

In [5] a continuous finite element method for the equation $\beta \cdot \nabla p=f$ was applied and a local error estimate $\left\|p-p_{h}\right\|_{0} \leq C h^{m+\frac{1}{4}}\|f\|_{m+1}$ was obtained. In [7] a streamline diffusion method for $\beta \cdot \nabla p+p=f$ was applied and the error estimates $\left\|p-p_{h}\right\|_{0} \leq C h^{m+\frac{1}{2}}\|p\|_{m+1}$ and $\left\|\beta \cdot \nabla\left(p-p_{h}\right)\right\|_{0} \leq C h^{m}\|p\|_{m+1}$ were obtained. Recently, in [8] a finite element method for the compressible Stokes problem has been studied, and the unique solvability, stability of its method was shown by putting the compressible Stokes problem into the framework of an abstract formulation. The following non-optimal error estimates were obtained:

$$
\left\|\mathbf{u}-\mathbf{u}_{h}\right\|_{1} \leq K h^{k}\left(\|\mathbf{f}\|_{k-1}+\|g\|_{k}\right)+K h^{m}\left(\|\mathbf{f}\|_{m}+\|g\|_{m+1}\right)
$$

and

$$
\left\|p-p_{h}\right\|_{0} \leq K h^{m}\left(\|\mathbf{f}\|_{m}+\|g\|_{m+1}\right)
$$

for some constant $K$. 
We improve this result for (1) using the streamline diffusion method. We here obtain the following main result. The proof is given in $\S 2$. Let $[\mathbf{u}, p]$ be a true solution of $(1)$ and $\left[\mathbf{u}_{h}, p_{h}\right]$ its finite element solution.

Theorem 1 Let $\epsilon>0$ be any number. Assume that $\mu$ is large enough or that the derivatives of $\rho \mathbf{U}$ and $\kappa \mathbf{U}$ are small enough in a suitable sense, then there is a constant $K$, not depending on $h$, such that

$$
\begin{aligned}
& \left\|\mathbf{u}-\mathbf{u}_{h}\right\|_{1}+h^{\epsilon / 2}\left\|\left(p-p_{h}\right)_{\beta} \sqrt{\kappa}\right\|_{0}+\left\|p-p_{h}\right\|_{0} \\
& \leq K \inf \left\{\|\nabla(\mathbf{u}-\hat{\mathbf{u}})\|_{0}+h^{\epsilon / 2}\left\|(p-\hat{p})_{\beta} \sqrt{\kappa}\right\|_{0}+h^{-\epsilon / 2}\|p-\hat{p}\|_{0}\right\},
\end{aligned}
$$

where the infimum is taken over all $\hat{\mathbf{u}} \in \mathbf{V}_{h}$ and $\hat{p} \in Q_{h}$.

Note that the inequality in (2) can be obtained for the case of the non-zero boundary value of $\mathbf{U}$ (see $\S 3$ and [9]).

In this paper, the following Sobolev spaces and norms are used: $H^{k}(\Omega)$, $k$ integer, $0 \leq k \leq \infty$, is the spaces of real valued $L^{2}$ functions on $\Omega$ such that all their derivatives up to order $k$ belong to $L^{2}(\Omega)$; the norm in $L^{2}$ is denoted by $\|u\|_{0}$ and $\|u\|_{k} \equiv \sqrt{\sum_{j=0}^{k}\left\|\nabla^{j} u\right\|_{0}^{2}}$ denotes that of $H^{k}$; also $H_{0}^{1}(\Omega) \equiv\left\{u \in H^{1}(\Omega):\left.u\right|_{\Gamma}=0\right\}, H_{0}^{k}(\Omega) \equiv H^{k}(\Omega) \cap H_{0}^{1}(\Omega) .|u|_{\infty} \equiv$ $\max \{|u(x)|: x \in \Omega\}$, and $L^{\infty}=\left\{u:|u|_{\infty}<\infty\right\}$.

Throughout this paper the following notation shall be used: the $L^{2}$ - inner product is $(f, g) \equiv \int_{\Omega} f g \mathrm{dx}$; and the derivative in the $\mathbf{U}$ direction is $\chi_{\beta} \equiv$ $\mathbf{U} \cdot \nabla \chi$ and $\|\chi\|_{Q} \equiv\left(\|\chi\|_{0}^{2}+\left\|\chi_{\beta} \sqrt{\kappa}\right\|_{0}^{2}\right)^{\frac{1}{2}}$. 
In our proofs $C$ denotes a generic constant, depending on certain quantities. We shall make this dependence explicitly, for example, writing $C(\Omega)$ if $C$ depends only on $\Omega$ (for example in the Sobolev inequalities), or $C\left(\Omega, \mathbf{u}_{0}, C_{0}\right)$ if $C$ depends both on $\Omega, \mathbf{u}_{0}$ and $C_{0}$, and so on.

\section{The case with $\mathrm{U}=0$ on $\Gamma$}

In this section we study and apply a streamline diffusion method for the system (1) and show an unique existence of the solution of its finite element method, and finally give an error estimate. To do this, we define several bilinear forms in order to formulate the system (1) into a discrete version. We let $\mathbf{V}=H_{0}^{1}(\Omega) \times H_{0}^{1}(\Omega)$, and $M \equiv L_{0}^{2}(\Omega)=$ the set of functions $\chi \in L^{2}(\Omega)$ which satisfy $\int_{\Omega} \chi \mathrm{dx}=0$ and $Q=\left\{\chi \in L_{0}^{2}(\Omega):\|\chi\|_{Q}<\infty\right\}$. We now define the following bilinear forms $a, b$ and $c$ by

$$
\begin{aligned}
a(\mathbf{u}, \mathbf{v}) & =\int_{\Omega}\left(\mu \nabla \mathbf{u} \cdot \nabla \mathbf{v}+\nu \operatorname{div} \mathbf{u} \operatorname{div} \mathbf{v}+\rho \mathbf{u}_{\beta} \mathbf{v}\right) \mathrm{dx}, \quad \mathbf{u}, \mathbf{v} \in \mathbf{V} \\
b(\mathbf{v}, p) & =\int_{\Omega} p \operatorname{div} \mathbf{v} \mathrm{dx}, \quad \mathbf{v} \in \mathbf{V} \text { and } p \in M \\
c(p, \chi) & =\int_{\Omega} \kappa p_{\beta} \chi \mathrm{dx}, \quad p \in Q \text { and } \chi \in M
\end{aligned}
$$

and also define

$$
\langle\mathbf{f}, \mathbf{v}\rangle=\int_{\Omega} \mathbf{f} \mathbf{v} \mathrm{dx}
$$




$$
\langle g, \chi\rangle=\int_{\Omega} \rho^{-1} g \chi \mathrm{dx} .
$$

Using these, a weak formulation of (1) is defined by: find $[\mathbf{u}, p] \in \mathbf{V} \times Q$ such that

$$
\begin{array}{ll}
a(\mathbf{u}, \mathbf{v})-b(\mathbf{v}, p)=\langle\mathbf{f}, \mathbf{v}\rangle, & \forall \mathbf{v} \in \mathbf{V}, \\
c(p, \chi)+b(\mathbf{u}, \chi)=\langle g, \chi\rangle, & \forall \chi \in M .
\end{array}
$$

It is shown in $[3,14]$ that if $g \in L_{0}^{2}(\Omega)$ and $\mathbf{U} \in H_{0}^{3}(\Omega) \times H_{0}^{3}(\Omega)$, and if $\|\mathbf{U}\|_{3}$ is small enough, problem (3) has a unique solution $[\mathbf{u}, p] \in \mathbf{V} \times Q$ which satisfies the a priori estimate: $\|\mathbf{u}\|_{3}+\|p\|_{2} \leq K\left(\|\mathbf{f}\|_{1}+\|g\|_{2}\right)$ for a constant $K$.

Let $h$ be a mesh size in a given triangulation $\mathcal{T}_{h}$ or an approximation indicator. We consider finite dimensional subspaces $\mathbf{V}_{h} \subset \mathbf{V}$ and $Q_{h} \subset Q$. We are going to approximate the problem (3) with extra streamline diffusion added to the continuity equation $(3)_{(b)}$. Hence the approximate problem, which is a streamline diffusion method for (3), is: for $\epsilon>0$, find $\left[\mathbf{u}_{h}, p_{h}\right] \in$ $\mathbf{V}_{h} \times Q_{h}$ such that

$$
\begin{aligned}
& a\left(\mathbf{u}_{h}, \mathbf{v}\right)-b\left(\mathbf{v}, p_{h}\right)=\langle\mathbf{f}, \mathbf{v}\rangle, \quad \forall \mathbf{v} \in \mathbf{V}_{h}, \\
& c\left(p_{h}, \chi+h^{\epsilon} \chi_{\beta}\right)+b\left(\mathbf{u}_{h}, \chi+h^{\epsilon} \chi_{\beta}\right)=\left\langle g, \chi+h^{\epsilon} \chi_{\beta}\right\rangle, \quad \forall \chi \in Q_{h} .
\end{aligned}
$$

In the next lemma we show the unique existence of the solution for the approximate problem (4). To do this, we cite the following inequality given in [8]: there is a constant $C$ such that

$$
\|\chi\|_{0} \leq C\|\nabla \chi\|_{-1}, \quad \forall \chi \in L_{0}^{2}(\Omega)
$$


where $\|\cdot\|_{-1}$ is a negative Sobolev norm. We let $\mu_{0}=\min \{\mu, \mu+\nu\}$ and $\gamma_{0}=$ $\frac{1}{2} \max \left\{|\operatorname{div}(\rho \mathbf{U})|_{\infty},|\operatorname{div}(\kappa \mathbf{U})|_{\infty}\right\}$ and shall denote $($,$) the inner product in$ the $L^{2}$ space.

Lemma 2 Let $\epsilon>0$ be any number. Assume that $\mu$ is large enough or that $\gamma_{0}$ is small enough, then the approximate problem (4) has a unique solution $\left[\mathbf{u}_{h}, p_{h}\right] \in \mathbf{V}_{h} \times Q_{h}$ and its solution satisfies the inequality

$$
\left\|u_{h}\right\|_{1}+\left\|p_{h}\right\|_{0}+h^{\epsilon / 2}\left\|p_{h, \beta} \sqrt{\kappa}\right\|_{0} \leq C\left(\|\mathbf{f}\|_{-1}+\|\mathbf{f}\|_{0}+\|g\|_{0}\right),
$$

where $C$ is a constant not depending on the meshsize $h$.

Proof: Letting $\mathbf{v}=\mathbf{u}_{h}$ and $\chi=p_{h}$ in (4) and adding the resulting two equations, we have

$$
\begin{aligned}
& \mu\left\|\nabla \mathbf{u}_{h}\right\|_{0}^{2}+\nu\left\|\operatorname{div} \mathbf{u}_{h}\right\|_{0}^{2}+\left(\rho \mathbf{u}_{h, \beta}, \mathbf{u}_{h}\right)+\left(\kappa p_{h, \beta}, p_{h}\right)+h^{\epsilon}\left\|p_{h, \beta} \sqrt{\kappa}\right\|_{0}^{2} \\
& +h^{\epsilon}\left(\operatorname{div} \mathbf{u}_{h}, p_{h, \beta}\right)=\left\langle\mathbf{f}, \mathbf{u}_{h}\right\rangle+\left\langle\rho^{-1} g, p_{h}+h^{\epsilon} p_{h, \beta}\right\rangle .
\end{aligned}
$$

Now, since

$$
h^{\epsilon}\left(\operatorname{div} \mathbf{u}_{h}, p_{h, \beta}\right) \geq-\frac{1}{2} h^{\epsilon}\left(|\kappa|_{\infty}^{-1}\left\|\nabla \mathbf{u}_{h}\right\|_{0}^{2}+\left\|p_{h, \beta} \sqrt{\kappa}\right\|_{0}^{2}\right)
$$

and

$$
\left(\rho \mathbf{u}_{h, \beta}, \mathbf{u}_{h}\right)+\left(\kappa p_{h, \beta}, p_{h}\right) \geq-\gamma_{0}\left(\left\|\mathbf{u}_{h}\right\|_{0}^{2}+\left\|p_{h}\right\|_{0}^{2}\right)
$$


we get

$$
\begin{aligned}
& \left(\mu_{0}-\frac{1}{2} h^{\epsilon}|\kappa|_{\infty}^{-1}\right)\left\|\nabla \mathbf{u}_{h}\right\|_{0}^{2}+\left(\frac{1}{2}-\frac{\delta_{2} h^{\epsilon}}{4}\right) h^{\epsilon}\left\|p_{h, \beta} \sqrt{\kappa}\right\|_{0}^{2}-\gamma_{0}\left(\left\|\mathbf{u}_{h}\right\|_{0}^{2}+\left\|p_{h}\right\|_{0}^{2}\right) \\
& -\frac{1}{4} \delta_{2}\left\|p_{h}\right\|_{0}^{2} \leq \delta_{1}\left\|\mathbf{u}_{h}\right\|_{0}^{2}+C \delta_{1}^{-1}\|\mathbf{f}\|_{0}^{2}+C \delta_{2}^{-1}\|g\|_{0}^{2}, \quad \forall \delta_{1}, \delta_{2}>0
\end{aligned}
$$

where $C=C\left(\gamma_{0},|\rho|_{\infty}^{-1},|\kappa|_{\infty}^{-1}\right)$. Next using the inequality (5) and the first equation in (4), we have

$$
\begin{aligned}
\left\|p_{h}\right\|_{0} & \leq C\left\|\nabla p_{h}\right\|_{-1} \\
& \leq C \sup _{\substack{\mathbf{v} \in H_{0}^{1}(\Omega) \\
\|\mathbf{v}\|_{1}=1}}\left\{a\left(\mathbf{u}_{h}, \mathbf{v}\right)-\langle\mathbf{f}, \mathbf{v}\rangle\right\} \\
& \leq c_{1}\left(\left\|\nabla \mathbf{u}_{h}\right\|_{0}+\|\mathbf{f}\|_{-1}\right),
\end{aligned}
$$

where $c_{1}=C\left(|\mathbf{U}|_{\infty},|\rho|_{\infty}\right)$. Finally using the Poincaré inequality $\left\|\mathbf{u}_{h}\right\|_{0} \leq$ $\bar{C}\left\|\nabla \mathbf{u}_{h}\right\|_{0}$, (8) and (9), we obtain

$$
\begin{aligned}
m_{1}\left\|\nabla \mathbf{u}_{h}\right\|_{0}^{2}+m_{2} h^{\epsilon}\left\|p_{h, \beta} \sqrt{\kappa}\right\|_{0}^{2} \leq & {\left[C \delta_{1}^{-1}+c_{1}^{2}\left(\gamma_{0}+\delta_{2}\right)\right]\left(\|\mathbf{f}\|_{0}^{2}+\|\mathbf{f}\|_{-1}^{2}\right) } \\
& +C \delta_{2}^{-1}\|g\|_{0}^{2}, \quad \forall \delta_{1}, \delta_{2}>0
\end{aligned}
$$

where $m_{1}=\mu_{0}-\frac{1}{2} h^{\epsilon}|\kappa|_{\infty}^{-1}-\left(1+c_{1}^{2}\right) \gamma_{0}-\frac{1}{4} c_{1}^{2} \delta_{2}-\delta_{1} \bar{C}^{2}$ and $m_{2}=\frac{1}{2}(1-$ $\left.\frac{1}{2} \delta_{2} h^{\epsilon}\right)$. Since the numbers $\delta_{1}$ and $\delta_{2}$ are arbitrary, the constants $m_{1}$ and $m_{2}$ can be made a positive number with a small condition of $\gamma_{0}$ and $h$, or a large condition of $\mu_{0}$. Thus the inequality (6) follows from (9), (10) and the Poincaré inequality. A standard argument shows the unique existence of the solution. 
Let $[\mathbf{u}, p]$ and $\left[\mathbf{u}_{h}, p_{h}\right]$ be the solution of (3) and (4) respectively, and let $[\hat{\mathbf{u}}, \hat{p}] \in \mathbf{V}_{h} \times Q_{h}$. We also put $e_{\mathbf{u}}=\mathbf{u}-\mathbf{u}_{h}, e_{p}=p-p_{h}$ and $\hat{\mathbf{e}}_{\mathbf{u}}=\mathbf{u}-\hat{\mathbf{u}}$, $\hat{e}_{p}=p-\hat{p}$. We next obtain an error estimate for the approximate problem (4), using a large condition on $\mu$ or a small condition on $|\nabla \mathbf{U}|_{\infty}$.

Theorem 3 Let $\epsilon>0$. Assume that $\mu$ is large enough or that $\gamma_{0}$ is sufficiently small, then there is a constant $K$, not depending on $h$, such that

$$
\begin{aligned}
& \left\|\mathbf{u}-\mathbf{u}_{h}\right\|_{1}+h^{\epsilon / 2}\left\|\left(p-p_{h}\right)_{\beta} \sqrt{\kappa}\right\|_{0}+\left\|p-p_{h}\right\|_{0} \\
\leq & K \inf \left\{\|\nabla(\mathbf{u}-\hat{\mathbf{u}})\|_{0}+h^{\epsilon / 2}\left\|(p-\hat{p})_{\beta} \sqrt{\kappa}\right\|_{0}+h^{-\epsilon / 2}\|p-\hat{p}\|_{0}\right\},
\end{aligned}
$$

where the infimum is taken over all $\hat{\mathbf{u}} \in \mathbf{V}_{h}$ and $\hat{p} \in Q_{h}$.

Proof: The proof is based on the following equations for the error:

$$
\begin{aligned}
& a\left(\mathbf{e}_{\mathbf{u}}, \mathbf{v}\right)-b\left(e_{p}, \mathbf{v}\right)=0, \quad \forall \mathbf{v} \in \mathbf{V}_{h}, \\
& c\left(e_{p}, \chi+h^{\epsilon} \chi_{\beta}\right)+b\left(\mathbf{e}_{\mathbf{u}}, \chi+h^{\epsilon} \chi_{\beta}\right)=0, \quad \forall \chi \in Q_{h} .
\end{aligned}
$$

Taking $\mathbf{v}=\mathbf{e}_{\mathbf{u}}-\hat{\mathbf{e}}_{\mathbf{u}} \in \mathbf{V}_{h}$ and $\chi=e_{p}-\hat{e}_{p} \in Q_{h}$ in (12), we have

$$
\begin{aligned}
& a\left(\mathbf{e}_{\mathbf{u}}, \mathbf{e}_{\mathbf{u}}\right)-b\left(e_{p}, \mathbf{e}_{\mathbf{u}}\right)=a\left(\mathbf{e}_{\mathbf{u}}, \hat{\mathbf{e}}_{\mathbf{u}}\right)-b\left(e_{p}, \hat{\mathbf{e}}_{\mathbf{u}}\right), \\
& c\left(e_{p}, e_{p}+h^{\epsilon} e_{p, \beta}\right)+b\left(\mathbf{e}_{\mathbf{u}}, e_{p}+h^{\epsilon} e_{p, \beta}\right) \\
& =c\left(e_{p}, \hat{e}_{p}+h^{\epsilon} \hat{e}_{p, \beta}\right)+b\left(\mathbf{e}_{\mathbf{u}}, \hat{e}_{p}+h^{\epsilon} \hat{e}_{p, \beta}\right) .
\end{aligned}
$$


Using (5) and (12) $a$ we have $\left\|e_{p}\right\|_{0} \leq c_{1}\left\|\nabla \mathbf{e}_{\mathbf{u}}\right\|_{0}$, and computing (13) we have

$$
\begin{aligned}
& \mu_{0}\left\|\nabla \mathbf{e}_{\mathbf{u}}\right\|_{0}^{2}+h^{\epsilon}\left\|e_{p, \beta} \sqrt{\kappa}\right\|_{0}^{2}-\frac{1}{2}|\operatorname{div}(\rho \mathbf{U})|_{\infty}\left\|\mathbf{e}_{\mathbf{u}}\right\|_{0}^{2} \\
& +c\left(e_{p}, e_{p}\right)+b\left(\mathbf{e}_{\mathbf{u}}, h^{\epsilon} e_{p, \beta}\right) \\
\leq & C\left(\left\|\nabla \mathbf{e}_{\mathbf{u}}\right\|\left\|_{0}\right\| \nabla \hat{\mathbf{e}}_{\mathbf{u}}\left\|_{0}+\right\| \nabla \mathbf{e}_{\mathbf{u}}\left\|_{0}\right\| \hat{\mathbf{e}}_{\mathbf{u}}\left\|_{0}+\right\| e_{p}\left\|_{0}\right\| \operatorname{div} \hat{\mathbf{e}}_{\mathbf{u}} \|_{0}\right) \\
& +\left\|e_{p, \beta} \sqrt{\kappa}\right\|_{0}\left\|\hat{e}_{p} \sqrt{\kappa}\right\|_{0}+h^{\epsilon}\left\|e_{p, \beta} \sqrt{\kappa}\right\|_{0}\left\|\hat{e}_{p, \beta} \sqrt{\kappa}\right\|_{0} \\
& +\left\|\operatorname{div} \mathbf{e}_{\mathbf{u}}\right\|_{0}\left(\left\|\hat{e}_{p}\right\|_{0}+|\kappa|_{\infty}^{-\frac{1}{2}} h^{\epsilon}\left\|\hat{e}_{p, \beta} \sqrt{\kappa}\right\|_{0}\right)
\end{aligned}
$$

(by Poincaré inequality)

$$
\begin{aligned}
\leq & C\left\|\nabla \mathbf{e}_{\mathbf{u}}\right\|_{0}\left(\left\|\nabla \hat{\mathbf{e}}_{\mathbf{u}}\right\|_{0}+h^{\epsilon}\left\|\hat{e}_{p, \beta} \sqrt{\kappa}\right\|_{0}+\left\|\hat{e}_{p}\right\|_{0}\right) \\
& +h^{\epsilon / 2}\left\|e_{p, \beta} \sqrt{\kappa}\right\|_{0}\left(h^{-\epsilon / 2}\left\|\hat{e}_{p} \sqrt{\kappa}\right\|_{0}+h^{\epsilon / 2}\left\|\hat{e}_{p, \beta} \sqrt{\kappa}\right\|_{0}\right) \\
\leq & C\left(\left\|\nabla \mathbf{e}_{\mathbf{u}}\right\|_{0}+h^{\epsilon / 2}\left\|e_{p, \beta} \sqrt{\kappa}\right\|_{0}\right) \times \\
& \left(\left\|\nabla \hat{\mathbf{e}}_{\mathbf{u}}\right\|_{0}+h^{\epsilon / 2}\left\|\hat{e}_{p, \beta} \sqrt{\kappa}\right\|_{0}+h^{-\epsilon / 2}\left\|\hat{e}_{p}\right\|_{0}\right),
\end{aligned}
$$

where $C=C\left(\bar{C},|\kappa|_{\infty}^{-1 / 2}\right)$. We also have

$$
\begin{aligned}
& c\left(e_{p}, e_{p}\right)+b\left(\mathbf{e}_{\mathbf{u}}, h^{\epsilon} e_{p, \beta}\right) \\
\geq & -\gamma_{0}\left\|e_{p}\right\|_{0}^{2}-\frac{h^{\epsilon}}{2}\left(|\kappa|_{\infty}^{-1}\left\|\operatorname{div} \mathbf{e}_{\mathbf{u}}\right\|_{0}^{2}+\left\|e_{p, \beta} \sqrt{\kappa}\right\|_{0}^{2}\right) \\
\geq & -\gamma_{0} c_{1}^{2}\left\|\nabla \mathbf{e}_{\mathbf{u}}\right\|_{0}^{2}-\frac{h^{\epsilon}}{2}\left(|\kappa|_{\infty}^{-1}\left\|\nabla \mathbf{e}_{\mathbf{u}}\right\|_{0}^{2}+\left\|e_{p, \beta} \sqrt{\kappa}\right\|_{0}^{2}\right) .
\end{aligned}
$$


Using (14) and (15) one easily obtains

$$
\begin{aligned}
k_{1}\left\|\nabla \mathbf{e}_{\mathbf{u}}\right\|_{0}^{2}+\frac{1}{2} h^{\epsilon}\left\|e_{p, \beta} \sqrt{\kappa}\right\|_{0}^{2} \leq & C\left(\left\|\nabla \mathbf{e}_{\mathbf{u}}\right\|_{0}+h^{\epsilon / 2}\left\|e_{p, \beta} \sqrt{\kappa}\right\|_{0}\right) \times \\
& \left(\left\|\nabla \hat{\mathbf{e}}_{\mathbf{u}}\right\|_{0}+h^{\epsilon / 2}\left\|\hat{e}_{p, \beta} \sqrt{\kappa}\right\|_{0}+h^{-\epsilon / 2}\left\|\hat{e}_{p}\right\|_{0}\right)
\end{aligned}
$$

where $k_{1}=\mu_{0}-\left(c_{1}^{2}+\bar{C}^{2}\right) \gamma_{0}-\frac{1}{2} h^{\epsilon}|\kappa|_{\infty}^{-1}$. The constant $k_{1}$ can be made a positive number by assuming that $\gamma_{0}$ and $h$ are small enough or that $\mu_{0}$ is large enough. Again rescaling the constants $k_{1}$ and $\frac{1}{2} h^{\epsilon}$ in (16) and using $(a+b)^{2} \leq 2\left(a^{2}+b^{2}\right)$ we obtain

$$
\left\|\nabla \mathbf{e}_{\mathbf{u}}\right\|_{0}+h^{\epsilon / 2}\left\|e_{p, \beta} \sqrt{\kappa}\right\|_{0} \leq C\left(\left\|\nabla \hat{\mathbf{e}}_{\mathbf{u}}\right\|_{0}+h^{\epsilon / 2}\left\|\hat{e}_{p, \beta} \sqrt{\kappa}\right\|_{0}+h^{-\epsilon / 2}\left\|\hat{e}_{p}\right\|_{0}\right) .
$$

Since $\hat{\mathbf{u}} \in \mathbf{V}_{h}$ and $\hat{p} \in Q_{h}$ were arbitrarily chosen, and using $\left\|e_{p}\right\|_{0} \leq$ $C\left\|\nabla \mathbf{e}_{\mathbf{u}}\right\|_{0}$, our inequality (11) easily follows.

We assume the following approximation properties (see $[6,4])$ :

$$
\begin{aligned}
& \inf _{\mathbf{v} \in \mathbf{V}_{h}}\|\mathbf{u}-\mathbf{v}\|_{1} \leq C h^{k}\|\mathbf{u}\|_{k+1}, \quad \forall \mathbf{u} \in \mathbf{V} \cap\left(H^{k+1}\right)^{2} \\
& \inf _{q \in Q_{h}}\|p-q\|_{0} \leq C h^{m}\|p\|_{m}, \quad \forall p \in Q \cap H^{m}
\end{aligned}
$$

Using (18) and Theorem 3 gives the following error bounds:

Theorem 4 Let $\epsilon>0$ be a number. Let $[\mathbf{u}, p]$ and $\left[\mathbf{u}_{h}, p_{h}\right]$ be the solutions of (3) and (4) respectively. suppose that $\mu$ is large enough or $\gamma_{0}$ is sufficiently 
small, and that $\mathbf{f} \in H^{l-1} \times H^{l-1}, g \in H^{l}$, where $l=\max \{k-1, m\}$ with $k \geq 1$ and $m \geq 1$, then there is a constant $K$, not depending on $h$, such that

$$
\begin{aligned}
\left\|\mathbf{u}-\mathbf{u}_{h}\right\|_{1}+\left\|p-p_{h}\right\|_{0} \leq K & {\left[h^{k}\left(\|\mathbf{f}\|_{k-1}+\|g\|_{k}\right)\right.} \\
& \left.+h^{m-\epsilon / 2}\left(\|\mathbf{f}\|_{m-1}+\|g\|_{m}\right)\right], \\
\left\|\left(p-p_{h}\right)_{\beta} \sqrt{\kappa}\right\|_{0} \leq K & {\left[h^{k-\epsilon / 2}\left(\|\mathbf{f}\|_{k-1}+\|g\|_{k}\right)\right.} \\
& \left.+h^{m-\epsilon}\left(\|\mathbf{f}\|_{m-1}+\|g\|_{m}\right)\right] .
\end{aligned}
$$

Proof: The proof easily follows from (11), (18) and the regularity result of the problem (1) (see $[3,14])$.

Remark. Consequently our error bound gives an order of accuracy $1-\epsilon / 2$ $(\epsilon>0)$ greater than that given in [8]. The theory given in this section may be possibly extended and applied to a bounded domain in $R^{n}$. For an example of the finite element subspaces $\mathbf{V}_{h}$ and $Q_{h}$, the MINI elements may be considered, i.e. the continuous piecewise linear plus cubic bubble functions for velocity and the continuous piecewise linear functions for pressure (see $[8])$. 


\section{Incoming compressible flow}

In this section we assume that the first component of the given vector field $\mathbf{U}=[U, V]$ is positive, in other words $U \geq C_{0}>0$ for a constant $C_{0}$. So the ambient flow moves from left to right and an inflow boundary condition is imposed on the pressure in the (hyperbolic) continuity equation (for details see [10]). Let $\mathbf{n}$ be the unit outward pointing normal vector on the boundary of the domain $\Omega$. The incoming and outgoing portions of the boundary are defined by

$$
\begin{gathered}
\Gamma_{\text {in }}=\{\mathbf{x} \in \Gamma: \mathbf{U} \cdot \mathbf{n}<0\}, \\
\Gamma_{\text {out }}=\{\mathbf{x} \in \Gamma: \mathbf{U} \cdot \mathbf{n} \geq 0\} .
\end{gathered}
$$

The equations to be considered become

$$
\left\{\begin{array}{l}
-\mu \Delta \mathbf{u}-\nu \nabla \operatorname{div} \mathbf{u}+\rho(\mathbf{U} \cdot \nabla) \mathbf{u}+\nabla p=\mathbf{f} \quad \text { in } \Omega \\
\operatorname{div} \mathbf{u}+\kappa \mathbf{U} \cdot \nabla p=\rho^{-1} g \quad \text { in } \Omega \\
\mathbf{u}=0 \quad \text { on } \Gamma \\
p=0 \quad \text { on } \Gamma_{\text {in }} .
\end{array}\right.
$$

For the existence and regularity of the solution $[\mathbf{u}, p]$ of $(20)$ see $[10$, Theorem 2.1].

The Banach spaces to be considered here are $\mathbf{V}=H_{0}^{1}(\Omega) \times H_{0}^{1}(\Omega), M=$ $L^{2}(\Omega)$ and $Q=\left\{\chi \in M:\|\chi\|_{Q}<\infty,\left.\chi\right|_{\Gamma_{i n}}=0\right\}$.

We note that a convergence analysis is not known for the corresponding approximate problem of the problem (20), i.e. find $\left[\mathbf{u}_{h}, p_{h}\right] \in \mathbf{V}_{h} \times Q_{h}$ 
satisfying

$$
\begin{array}{ll}
a\left(\mathbf{u}_{h}, \mathbf{v}\right)-b\left(\mathbf{v}, p_{h}\right)=\langle\mathbf{f}, \mathbf{v}\rangle, & \forall \mathbf{v} \in \mathbf{V}_{h}, \\
c\left(p_{h}, \chi\right)+b\left(\mathbf{u}_{h}, \chi\right)=\langle g, \chi\rangle, & \forall \chi \in M_{h},
\end{array}
$$

where $\mathbf{V}_{h} \subset \mathbf{V}, Q_{h} \subset Q$ and $M_{h} \subset M$ are the finite dimensional subspaces, and so we apply to (20) the streamline diffusion formulation (4) and can show a similar convergence result like the ones in (19). On the other hand, it is shown in $[11,12]$ that a different weak formulation of the continuity equation for (20) gives a convergence analysis under suitable conditions.

The following inequality (22) is comparable with (5) and can be easily shown by following the same procedures as those in [10, p.100], in which the $L^{2}$ norm of pressure is bounded by the one of the streamline (convective) derivative, that is,

$$
\|\chi\|_{0} \leq C\left(\Omega, C_{0}\right)\left\|\chi_{\beta}\right\|_{0}, \quad(\chi \in Q) .
$$

Now choosing finite element subspaces $\mathbf{V}_{h} \subset \mathbf{V}$ and $Q_{h} \subset Q$, and using (22), and applying the same procedures as those in $\S 2$ to the equations in (20), one can easily obtain the same results like Lemma 2, Theorem 3 and 4 under the similar hypotheses.

As an example of the finite element subspaces, it is assumed that there is a triangulation $\mathcal{T}_{h}=\{T\}$, which is a standard finite element subdivision of $\Omega$ into nonoverlapping triangles $T$ of diameter $h_{T}$. Here, $h=$ $\max \left\{h_{T}: T \in \mathcal{T}_{h}\right\}$. Let $\mathcal{P}(T)$ be the space of linear functions on $T$. Let 
$\mathbf{V}_{h}$ and $Q_{h}$ be defined by

$$
\begin{aligned}
& \mathbf{V}_{h}=\left\{\mathbf{u}_{h} \in \mathbf{V}: \mathbf{u}_{h} \text { continuous on } \Omega,\left.\mathbf{u}_{h}\right|_{T} \in(\mathcal{P}(T))^{2}, \forall T \in T_{h}\right\}, \\
& Q_{h}=\left\{p_{h} \in Q: p_{h} \text { continuous on } \Omega,\left.p_{h}\right|_{T} \in \mathcal{P}(T), \forall T \in T_{h}\right\} .
\end{aligned}
$$

The finite element spaces in (23) are used in the next examples.

\section{$4 \quad$ Numerical examples}

Numerical examples are given for the corresponding discrete problem (4) of the problem (20), using (22) as finite element subspaces. For the finite element program used here see [9]. An initial triangulation is started on a given domain and also curved triangles are used if the boundary is curved, and then refined triangulations are generated sequentially as the step increases. The midpoints of those edges having their two end points on the curved boundary are projected onto the curved boundary. On each triangulation we solve problem (4) by multiplying the transpose of a finite element matrix to itself and by applying to the resulting matrix the conjugate gradient method.

Here "nvar" denotes the number of unknowns approximately and "elts" denotes the number of triangles. The seven point quadrature rule in [13] is considered. In the examples, if $\rho(P)=P$, then $d \rho / d P=1$. If we take $P=1$, then $\rho=1, \kappa=1$. In our numerical examples the values of $\epsilon$ in (4) are taken to be $\epsilon=1$. 
Case 1. As our first example we use the exact solutions in [10] which describe the essential property of the problem (20) near the top point $(0,1)$ of the unit disk with centre 0 . Here the viscous constants were chosen $\mu=1$ and $\nu=0$, and $U=1, V=0$ were chosen. The functions to be tested in the finite element program are

$$
\begin{aligned}
& u(x, y)=1-x^{2}-y^{2}, \\
& v(x, y)=\frac{1}{2}(1+y)\left[\left(1-y^{2}\right)^{3 / 2}-|x|^{3}\right], \\
& p(x, y)=\frac{3}{2}(1+y)(x-1)\left(x+\sqrt{1-y^{2}}\right) .
\end{aligned}
$$

The functions $u, v$ and $p$ obviously satisfy their boundary conditions and do not have any singularity, but their derivatives $p_{y}$ and $v_{y y}$ have mild singularities near $(0,1)$ and the behaviour of $p_{y}$ is like the one of $v_{y y}$ there, but $p_{y y} \notin L^{q}(\Omega)$ for $2<q<3$. The right hand sides are

$$
\begin{aligned}
f_{1}= & 4+x(3 y+1)-\frac{3}{2}(y+1)\left(1-\sqrt{1-y^{2}}\right), \\
f_{2}= & 3|x|(1+y)-\frac{3}{2}(1+y) x|x|+\frac{3}{2} x(x-1) \\
& +\frac{3}{2}(x+3 y) \sqrt{1-y^{2}}+\frac{3}{2}(1-x-y)\left(y+y^{2}\right) \frac{1}{\sqrt{1-y^{2}}}
\end{aligned}
$$

and $g=2\left(1-y^{2}\right)^{\frac{3}{2}}-\frac{1}{2}|x|^{3}+x+3 y\left(x-\frac{1}{2}\right)-\frac{3}{2}$.

On next tables the errors in the $H^{1}$ norm velocity and $L^{2}, \beta$ norms of pressure are given, and Table 2 shows that they are converging linearly in the $H^{1}$ norm velocity and $L^{2}$ norm pressure but are not in the $\beta$ 
TABLE 1: The errors in streamline diffusion method

\begin{tabular}{|r|c|c|c|c|}
\hline nvar & elts & $\left\|\mathbf{u}-\mathbf{u}_{h}\right\|_{1}$ & $\left\|p-p_{h}\right\|_{0}$ & $\left\|\left(p-p_{h}\right)_{\beta}\right\|_{0}$ \\
\hline \hline 39 & 16 & 0.98527 & 0.15482 & 1.17492 \\
\hline 123 & 64 & 0.52861 & 0.08503 & 0.70499 \\
\hline 435 & 256 & 0.27154 & 0.03591 & 0.41408 \\
\hline 1635 & 1024 & 0.13695 & 0.01403 & 0.24734 \\
\hline 6339 & 4096 & 0.06870 & 0.00567 & 0.14848 \\
\hline 24963 & 16384 & 0.03440 & 0.00237 & 0.08948 \\
\hline
\end{tabular}

TABLE 2: The rate of convergence

\begin{tabular}{|c|c|c|c|}
\hline$*$ & $\left\|\mathbf{u}-\mathbf{u}_{h}\right\|_{1}$ & $\left\|p-p_{h}\right\|_{0}$ & $\left\|\left(p-p_{h}\right)_{\beta}\right\|_{0}$ \\
\hline \hline 1 & 1.08419 & 1.04344 & 0.88936 \\
2 & 1.05472 & 1.36481 & 0.84253 \\
3 & 1.03394 & 1.41961 & 0.77836 \\
4 & 1.01819 & 1.33721 & 0.75319 \\
5 & 1.00927 & 1.27280 & 0.73896 \\
\hline
\end{tabular}

norm pressure. The reason for this is that we do not have an optimal error estimate for the method (4).

Case 2. The next tables give numerical numbers for solving the same problem, using the method of [8] on the unit disk in Case 1. That is, in the place of the second equation of (4) we use the natural formulation $\left(\operatorname{div} \mathbf{u}_{h}, \chi\right)+\left(p_{h, \beta}, \chi\right)=\langle g, \chi\rangle$. In the $H^{1}$ norm velocity and $L^{2}$ norm 
TABLE 3: No streamline diffusion method

\begin{tabular}{|r|c|c|c|c|}
\hline nvar & elts & $\left\|\mathbf{u}-\mathbf{u}_{h}\right\|_{1}$ & $\left\|p-p_{h}\right\|_{0}$ & $\left\|\left(p-p_{h}\right)_{\beta}\right\|_{0}$ \\
\hline \hline 39 & 16 & 0.98793 & 0.44888 & 1.59449 \\
\hline 123 & 64 & 0.52678 & 0.12718 & 0.91553 \\
\hline 435 & 256 & 0.27063 & 0.07656 & 0.94176 \\
\hline 1635 & 1024 & 0.13704 & 0.05253 & 1.21296 \\
\hline 6339 & 4096 & 0.06890 & 0.03566 & 1.65043 \\
\hline
\end{tabular}

pressure the numbers are rather slowly converging to zero when compared with those in Table 1 , but the errors in the $\beta$ norm pressure are not converging at all. Note that a convergence analysis for this formulation is not known. On table 4 the exactly same problem was tested, using the bubble function for velocity. In this case $2 \times$ elts is added to the unknowns "nvar" roughly. It is observed that the numbers obtained are more accurate than those in Table 3 , but still the $\beta$ norm error for pressure is not converging.

Case 3. Next, the functions in (23) are considered on $\Omega=(-1,1) \times(-1,1)$, and the constants $\mu=1, \nu=0, U=1, V=0$ were chosen. So the discrete problem (4) is solved on each given triangulation, using the streamline diffusion method. Note that curved triangles are not used in the triangulation of $\Omega$. Even though an optimal error estimate for (4) was not mathematically established, the errors in Table 5, especially in the $\beta$ norm, show nearly an $\mathcal{O}(h)$ convergence in Table 6 , when comparing to the rates of convergence in Table 2. 
TABLE 4: No streamline diffusion method by bubble functions.

\begin{tabular}{|r|c|c|c|c|}
\hline nvar & elts & $\left\|\mathbf{u}-\mathbf{u}_{h}\right\|_{1}$ & $\left\|p-p_{h}\right\|_{0}$ & $\left\|\left(p-p_{h}\right)_{\beta}\right\|_{0}$ \\
\hline \hline 66 & 16 & 0.39436 & 0.36659 & 0.53128 \\
\hline 242 & 64 & 0.21602 & 0.10903 & 0.32857 \\
\hline 930 & 256 & 0.11129 & 0.06201 & 0.32339 \\
\hline 3650 & 1024 & 0.05635 & 0.03789 & 0.37328 \\
\hline 14466 & 4096 & 0.02831 & 0.02071 & 0.40301 \\
\hline
\end{tabular}

TABLE 5: The errors in the rectangle domain

\begin{tabular}{|r|c|c|c|c|}
\hline nvar & elts & $\left\|\mathbf{u}-\mathbf{u}_{h}\right\|_{1}$ & $\left\|p-p_{h}\right\|_{0}$ & $\left\|\left(p-p_{h}\right)_{\beta}\right\|_{0}$ \\
\hline \hline 70 & 32 & 1.22393 & 0.75447 & 1.33933 \\
\hline 234 & 128 & 0.66891 & 0.31704 & 0.75439 \\
\hline 850 & 512 & 0.35458 & 0.14181 & 0.40848 \\
\hline 3234 & 2048 & 0.18502 & 0.06782 & 0.21646 \\
\hline 12610 & 8192 & 0.09572 & 0.03345 & 0.11348 \\
\hline 49794 & 32768 & 0.04919 & 0.01585 & 0.05916 \\
\hline
\end{tabular}


TABLE 6: The rate of convergence

\begin{tabular}{|c|c|c|c|}
\hline$*$ & $\left\|\mathbf{u}-\mathbf{u}_{h}\right\|_{1}$ & $\left\|p-p_{h}\right\|_{0}$ & $\left\|\left(p-p_{h}\right)_{\beta}\right\|_{0}$ \\
\hline \hline 1 & 1.00126 & 1.43681 & 0.95128 \\
2 & 0.98412 & 1.24743 & 0.95117 \\
3 & 0.97358 & 1.10404 & 0.95048 \\
4 & 0.96862 & 1.03883 & 0.94914 \\
5 & 0.96947 & 1.08764 & 0.94856 \\
\hline
\end{tabular}

Acknowledgement: The author expresses sincere gratitude to Professor R. Bruce Kellogg for suggesting this direction. The author also thanks Professor J. Vanden-Broeck for numerous comments and advice. The author acknowledge the financial support of the Korea Research Foundation made in the program year of 1998.

\section{References}

[1] R. A. Adams, Sobolev Spaces, Academic press, New York, 1975.

[2] D. N. Arnold, F. Brezzi, M. Fortin, A stable finite element for the Stokes equations, Calcolo 21, 337-344 (1984).

[3] H. Beirāo da Veiga, Stationary motions and the incompressible limit for compressible viscous fluids, Houston J. of Math., 13 (1987), 527-544. 
[4] P. G. Ciarlet, The Finite Element Method for Elliptic Problems, NorthHolland, 1978.

[5] R. S. Falk, G. R. Richter, Local error estimates for a finite element method for hyperbolic and convection-diffusion equations, SIAM J. Numer. Anal., 29 (1992), 730-754.

[6] V. Girault, P. A. Raviart, Finite Element Methods for Navier-Stokes Equations: Theory and Algorithms, Springer Series in Computational Mathematics 5 (1986).

[7] C. Johnson, Numerical solution of partial differential equations by the finite element method, Cambridge, 1987.

[8] R. B. Kellogg, B. Liu, A finite element method for the compressible Stokes equations, SIAM J. Numer. Anal., 1 (1993), 780-788.

[9] J. R. Kweon, Compressible Navier-Stokes Equations in two dimensions with inflow boundary condition and Numerical Experiments, Ph.D thesis (University of Maryland, College Park, 1995).

[10] J. R. Kweon, R. B. Kellogg, Compressible Navier-Stokes Equations in a bounded domain with Inflow Boundary Condition, SIAM J. Math. Anal., 28 (1997), 94-108.

[11] J. R. Kweon, Finite Element methods for Compressible Stokes Equations with Inflow Boundary Condition, Bull. Austral. Math. Soc., 56 (1997), 217-225. 
[12] J. R. Kweon, An optimal order convergence for a weak formulation of the compressible Stokes system with inflow boundary condition, Numer. Math., (2000).

[13] J. N. Lyness, D. Jesperson, Moderate degree symmetric quadrature rules for the triangle, Journal of the Institute of Mathematics and its Applications, 15 (1975), 19-32.

[14] A. Valli, On the existence of stationary solutions to compressible NavierStokes equations, Analyse non linéaire 4 (1987), 99-113. 